\title{
SISTEMA GENERAL DE REGALÍAS: NUEVOS RECURSOS PARA LA CIENCIA, TECNOLOGÍA E INNOVACIÓN EN COLOMBIA
}

\author{
General system of royalties: new resources for science, technology and innovation in \\ Colombia

\section{Yiseth Marina Becerra Arévalo} \\ Economista por la Universidad de Antioquia. Funcionaria de Colciencias. Bogotá, Colombia. \\ yisethmar@gmail.com
}

\begin{abstract}
Cómo citar /How to cite
Becerra, Y. (2015). Sistema general de regalías: nuevos recursos para la ciencia, tecnología e innovación en Colombia. Revista CEA, 1(1), 75-91.
\end{abstract}

Recibido: 4 de junio de 2014

Aceptado: 17 de julio de 2014

Resumen

Este artículo presenta un balance de la implementación del Fondo de Ciencia, Tecnología e Innovación del Sistema General de Regalías en Colombia (FCTel-SGR). La entrada en vigencia del SGR y la respectiva creación del FCTel-SGR, ha abierto una oportunidad para la ciencia, la tecnología y la innovación en el país y se espera que estos nuevos recursos impacten a las regiones. Antioquia se destaca por el número de proyectos aprobados y la diversificación de temas estratégicos priorizados para presentar al FCTel-SGR, enfocando los esfuerzos en el fortalecimiento de la CTel en los sectores de la salud, agropecuario y formación de capital humano, entre otros.

Palabras clave: sistema general de regalías, ciencia, tecnología, innovación.

\begin{abstract}
This paper presents an overview of the implementation of the Fund for Science, Technology and Innovation of General system royalties in Colombia (FCTel-SGR). The entry into force of the SGR and the ensuing creation of FCTel-SGR has brought an opportunity for Science, Technology and Innovation in the country and these new resources are expected to have an impact on the regions not only in the short, but also in the mid and long term. Antioquia stands out due to the number of projects approved and the diversification of prioritized strategic issues presented to FCTel-SGR, focusing efforts on strengthening the STI in the sectors of health, agriculture and human capital formation, among others.
\end{abstract}

Keywords: general system royalties, science, technology, innovation. 


\section{INTRODUCCIÓN}

Este artículo presenta un balance de la implementación del Fondo de Ciencia, Tecnología e Innovación del Sistema General de Regalías en Colombia (FCTel-SGR). La entrada en vigencia del SGR y la respectiva creación del FCTel-SGR, ha abierto una oportunidad para la ciencia, la tecnología y la innovación en el país, y se espera que estos nuevos recursos impacten a las regiones no solo en el corto sino también en el mediano y largo plazo. Las regiones han orientado su estrategia de inversión a atender problemas específicos a través de actividades de investigación e innovación, así como a la formación de capital humano, capital que podrá posteriormente elevar las capacidades de investigación e innovación en las regiones. Por su parte, Antioquia se ha destacado en el número de proyectos aprobados y en la diversificación de temas estratégicos priorizados para presentar al FCTel-SGR, enfocando los esfuerzos en el fortalecimiento de la CTel en los sectores de la salud, agropecuario y formación de capital humano, entre otros.

El artículo presenta, luego de esta introducción, una caracterización del nuevo Sistema General de Regalías, señalando algunas diferencias con el enfoque anterior. En el tercer punto, se presentan especialmente, resultados relacionados con los programas nacionales de ciencia, tecnología e innovación, incluyendo un balance para Antioquia. Se concluye que la entrada en vigencia del SGR y la respectiva creación del Fondo de CTel han abierto una oportunidad para este en el país.

\section{EL NUEVO SISTEMA GENERAL DE REGALÍAS}

Las regalías son la contraprestación económica que recibe el Estado por la explotación de un recurso natural no renovable, cuya producción se extingue por el transcurso del tiempo (DNP, 2007, p. 11). Hasta el 2011, en el antiguo esquema de regalías existían dos tipos: i) Directas: aquellas asignadas entre municipios $y$ departamentos en los que se explotaban recursos naturales no renovables $(80 \%)$, estos recursos debían destinarse a alcanzar las metas de coberturas para financiar los proyectos priorizados incluidos en los planes de desarrollo ii) Indirectas: aquellas no asignadas directamente a los municipios y departamentos productores, cuya administración correspondía al Fondo Nacional de Regalías (20\%); estos recursos se destinaban a la promoción de la minería, medio ambiente, y a financiar proyectos regionales de inversión definidos como prioritarios en los planes de desarrollo.

El antiguo esquema de regalías presentó ineficiencias que llevaron al gobierno nacional a modificarlo, siendo las más importantes las siguientes (Ministerio de Minas y Energía, 2011):

- Inequidad y pobreza: la distribución de recursos de regalías generaba la concentración del $80 \%$ de estos recursos en entidades territoriales que representan el $17 \%$ de la población.

- Ineficiencia: de las entidades territoriales beneficiarias de recursos de regalías y compensaciones, ninguna había logrado cumplir todas las coberturas mínimas en los sectores de educación, afiliación a salud, mortalidad infantil, agua potable y alcantarillado.

- Incorrecta utilización de los recursos de regalías: en la vigencias 2005 a 2010 el DNP reportó 21.681 presuntas irregularidades a órganos de control.

Así, en 2011, el antiguo esquema de regalías asumió un cambio radical con la expedición del Acto Legislativo 005 de 2011, con el cual se modificaron los artículos 360 y 361 de la Constitución Nacional y se creó el Sistema General de Regalías -SGR-. A través del acto 
legislativo se eliminó el sistema de asignaciones directas y quedó consagrado constitucionalmente un nuevo modelo de distribución y lineamientos generales sobre los cuales se estructuró el nuevo Sistema General de Regalías.

El nuevo SGR tiene como objetivos crear condiciones de equidad en la distribución de los ingresos que generen ahorros para épocas de escasez, distribuir los recursos hacia la población más pobre generando mayor equidad social, promover el desarrollo y la competitividad regional, incentivar proyectos minero energéticos (tanto para la pequeña, media industria y la minería artesanal), promover la integración de las entidades territoriales en proyectos comunes y propiciar la inversión en la restauración social y económica de los territorios donde se desarrollen actividades de exploración y explotación.

Para lograr los anteriores objetivos, el nuevo SGR organizó una distribución de los recursos teniendo en cuenta un mayor impacto en toda la población del país. Así, los ingresos del SGR se distribuirán de la siguiente manera: i) $10 \%$ para el Fondo de Ciencia, Tecnología e Innovación ii) 10\% para ahorro pensional territorial iii) hasta un $30 \%$ para el Fondo de Ahorro y Estabilización. Los recursos restantes se distribuirán en un $20 \%$ para las asignaciones directas, y un $80 \%$ para los Fondos de Compensación Regional y de Desarrollo Regional. También se destina un $2 \%$ para fiscalización de la exploración y explotación de los yacimientos, y el conocimiento y cartografía geológica del subsuelo.

Posteriormente se expidió la Ley 1530 del 17 de mayo del 2012, la cual reguló la organización y el funcionamiento del SGR, cuyo objeto es:
Determinar la distribución, objetivos, fines, administración, ejecución, control, el uso eficiente y la destinación de los ingresos provenientes de la explotación de los recursos naturales no renovables precisando las condiciones de participación de sus beneficiarios. Este conjunto de ingresos, asignaciones, órganos, procedimientos y regulaciones constituye el Sistema General de Regalías.

EI SGR está integrado por la Comisión Rectora, el Departamento Nacional de Planeación, los Ministerios de Hacienda y Crédito Público, y de Minas y Energía, Colciencias y los órganos colegiados de administración y decisión -OCAD-. La Comisión Rectora, es el órgano encargado de definir la política del SGR, evaluar su ejecución general y dictar regulación orientada a asegurar el adecuado funcionamiento del sistema. La Comisión Rectora está conformada por el Director del Departamento Nacional de Planeación -DNP; Ministro de Minas y Energía; Ministro de Hacienda y Crédito Público; dos gobernadores; dos alcaldes; un senador y un representante a la Cámara ( Art. 4 de la Ley 1530 de 2012).

Por su parte, los OCAD, son los responsables de evaluar, viabilizar, aprobar y priorizar la conveniencia y oportunidad de financiar los proyectos y/o programas. El OCAD del Fondo de Ciencia, Tecnología e Innovación está conformado por tres vértices: 1) Gobierno Nacional: Departamento Administrativo de Ciencia, Tecnología e Innovación, Colciencias; Departamento Nacional de Planeación, DNP; tres Ministerios (Ministerio de Tecnologías de la Información y las Comunicaciones, MinTIC, Ministerio de Educación Nacional y Ministerio de Agricultura y Desarrollo Rural) 2) gobierno departamental: seis gobernadores (uno por cada región), y 3) universidades: universidades públicas (3); universidades privadas (2); una universidad pública representante de comunidades étnicas. 
La Secretaría Técnica del OCAD del Fondo de Ciencia, Tecnología e Innovación está a cargo del Departamento Administrativo de Ciencia, Tecnología e Innovación -Colciencias, que se encarga de verificar el cumplimiento de los requisitos que establezca la Comisión Rectora del SGR para la aprobación de los programas o proyectos, y solicitar a expertos conceptos que den cuenta de la pertinencia técnica y científica de los programas y proyectos presentados al Fondo. Adicionalmente, Colciencias tiene un papel destacado en la formulación de políticas de CTel, para proponer a la Comisión Rectora del SGR los lineamientos de política que articulen el Sistema Nacional de Ciencia, Tecnología e Innovación con la política de inversión de recursos del Fondo CTel (Art. 29 Ley 1530 de 2012).

\section{EL FONDO DE CIENCIA, TECNOLOGÍA E INNOVACIÓN DEL SISTEMA GENERAL DE REGALÍAS - FCTel - SGR}

Como se planteó, con la expedición del nuevo SGR se crea el Fondo de Ciencia, Tecnología e Innovación, con una asignación del $10 \%$ del total de los recursos del sistema que benefician a todos los departamentos del país. El Ministerio de Hacienda y Crédito Público definió la fórmula de distribución de los recursos para los departamentos, teniendo en cuenta la población total, la tasa de desempleo y el Índice de Necesidades Básicas Insatisfechas (NBI).

EI FCTel-SGR tiene como finalidad,

\begin{abstract}
la financiación de proyectos regionales acordados entre las entidades territoriales y el Gobierno Nacional (Acto legislativo 05/11) y permitirá incrementar la capacidad científica, tecnológica, de innovación y de competitividad de las regiones, mediante proyectos que contribuyan a la producción, uso, integración y apropiación del conocimiento en el aparato productivo y en la sociedad en general, incluidos los proyectos relacionados con biotecnología y tecnologías de la información y las comunicaciones (Ley 1530 de 2012).
\end{abstract}

Las actividades que se financian con recursos del FCTel-SGR son i) Investigación y Desarrollo, ii) Innovación, iii) Formación y capacitación científica y tecnológica y iv) Servicios científicos y tecnológicos (Acuerdo 15/12). Así mismo, los proyectos deben ser pertinentes, coherentes y consistentes con los Planes Departamentales de Desarrollo y otros ejercicios de planeación estratégica regional (planes y agendas regionales de competitividad y los planes estratégicos departamentales de CTel, planes y estrategias sectoriales del orden nacional).

El Sistema General de Regalías destinó un presupuesto de $\$ 2.660 .566 .132 .711$ para el FCTel-SGR, de los cuales a marzo de 2014 se aprobaron \$1.549.481.897.025, por lo que queda un saldo de \$1.111.084.235.686. Desde la entrada en funcionamiento del nuevo SGR, y de acuerdo con información del Órgano Colegiado de Administración y Decisión -OCAD- del FCTel-SGR, a marzo de 2014 se aprobaron 204 proyectos.

La Región Caribe lidera la inversión en CTelcon un $30 \%$ del total de los recursos aprobados y le sigue el Eje Cafetero con una inversión del $17 \%$ del total (ver Tabla 1) 
Tabla 1. Total programas y proyectos aprobados por región Table 1. Total of programs and projects per region

\begin{tabular}{|l|c|c|c|c|c|}
\hline \multirow{2}{*}{\multicolumn{1}{c|}{ REGIÓN }} & & \multicolumn{2}{c|}{ Vr. FCTel } & \multirow{2}{*}{ CONTRAP. } & \multirow{2}{*}{ Vr. TOTAL } \\
\cline { 2 - 5 } & No. & VALOR & $\%$ & & \\
\hline Total general & $\mathbf{2 0 4}$ & $\mathbf{1 . 5 4 9 . 4 8 2}$ & $\mathbf{1 0 0 \%}$ & $\mathbf{4 4 5 . 4 1 0}$ & $\mathbf{1 . 9 9 4 . 8 9 2}$ \\
\hline Caribe & 41 & 458.251 & $30 \%$ & 56.344 & 514.594 \\
\hline Eje Cafetero & 42 & 270.492 & $17 \%$ & 148.809 & 419.301 \\
\hline Centro Oriente & 24 & 246.328 & $16 \%$ & 86.667 & 338.106 \\
\hline Pacífico & 38 & 242.389 & $16 \%$ & 62.281 & 302.660 \\
\hline Centro Sur & 36 & 192.714 & $12 \%$ & 37.132 & 229.845 \\
\hline Llanos & 23 & 139.309 & $9 \%$ & 54.177 & 190.385 \\
\hline
\end{tabular}

Fuente: Secretaría Técnica FCTel -SGR, Abril 01 de 2014 / Cifras en millones de pesos

Respecto a la asignación de los recursos por departamentos, se registra un total de 31 departamentos y Bogotá D.C, con proyectos y programas aprobados. Antioquia, Cauca, Cundinamarca, Huila y Tolima, han liderado la aprobación con $26,19,12,12$ y 11 proyectos respectivamente (ver Tabla 2). De igual manera, se destaca que estos departamentos son los que cuentan con mayores recursos apropiados para CTel según la distribución del Fondo CTel-SGR.

Tabla 2. Recursos apropiados y aprobados FCTel-SGR por departamento Table 2. Assigned and approved budgets FCTel-SGR per department

\begin{tabular}{|l|c|c|c|}
\hline \multicolumn{1}{|c|}{ DEPARTAMENTO } & $\begin{array}{c}\text { PROYECTOS } \\
\text { APROBADOS }\end{array}$ & TOTAL APROPIADO (\$) & TOTAL APROBADO (\$) \\
\hline Amazonas & $\mathbf{2 0 4}$ & $\mathbf{2 . 6 6 0 . 5 6 6}$ & $\mathbf{1 . 5 4 9 . 4 8 2}$ \\
\hline Antioquia & $\mathbf{2}$ & 24.948 & 12.612 \\
\hline Arauca & 26 & 180.530 & 175.781 \\
\hline Arch. San Andrés & 2 & 44.329 & 28.370 \\
\hline Atlántico & 8 & 24.662 & 0 \\
\hline Bogotá D.C & 5 & 83.268 & 49.975 \\
\hline Bolívar & 2 & 64.215 & 59.291 \\
\hline Boyacá & 1 & 151.280 & 45.014 \\
\hline Caldas & 8 & 103.795 & 2.175 \\
\hline Caquetá & 5 & 48.949 & 40.083 \\
\hline Casanare & 3 & 64.116 & 56.258 \\
\hline Cauca & 19 & 49.687 & 40.918 \\
\hline Cesar & 2 & 128.988 & 124.678 \\
\hline Chocó & 6 & 99.850 & 43.027 \\
\hline Córdoba & 10 & 90.939 & 160.284 \\
\hline Cundinamarca & 12 & 174.469 & 86.470 \\
\hline Guainía & 2 & 100.019 & 5.431 \\
\hline Guajira & 8 & 20.961 & 83.946 \\
\hline Guaviare & 4 & 121.024 & 11.268 \\
\hline Huila & 12 & 30.032 & 60.154 \\
\hline Magdalena & 4 & 97.309 & 26.783 \\
\hline Meta & 10 & 117.373 & 60.817 \\
\hline Nariño & 52.895 & \\
\hline
\end{tabular}




\begin{tabular}{|l|c|c|c|}
\hline \multicolumn{1}{|c|}{ DEPARTAMENTO } & $\begin{array}{c}\text { PROYECTOS } \\
\text { APROBADOS }\end{array}$ & TOTAL APROPIADO (\$) & TOTAL APROBADO (\$) \\
\hline \multicolumn{1}{|c|}{ TOTAL } & $\mathbf{2 0 4}$ & $\mathbf{2 . 6 6 0 . 5 6 6}$ & $\mathbf{1 . 5 4 9 . 4 8 2}$ \\
\hline Norte de Santander & 1 & 95.730 & 44.953 \\
\hline Putumayo & 1 & 53.007 & 10.787 \\
\hline Quindío & 3 & 21.999 & 15.283 \\
\hline Risaralda & 5 & 41.645 & 39.345 \\
\hline Santander & 5 & 80.715 & 53.440 \\
\hline Sucre & 7 & 109.350 & 61.015 \\
\hline Tolima & 11 & 79.254 & 52.903 \\
\hline Valle del Cauca & 3 & 108.135 & 12.948 \\
\hline Vaupés & 5 & 21.100 & 7.111 \\
\hline Vichada & 3 & 29.548 & 19.428 \\
\hline
\end{tabular}

Fuente: Ministerio de Hacienda y Crédito Público y Secretaría Técnica FCTel -SGR, Abril 01 de 2014 / Cifras en millones de pesos corrientes

\section{Resultados por Programas Nacionales de Ciencia, Tecnología e Innovación - PNCTel}

Los 204 programas y proyectos aprobados a la fecha se encuentra distribuidos en 14 áreas del conocimiento $\mathrm{o}$ programas especiales: agropecuarias; formación de alto nivel; programa Ondas; salud; electrónica, telecomunicaciones e informática; mar y recursos hidrobiológicos; centros y parques tecnológicos; ambiente, biodiversidad y hábitat; desarrollo tecnológico e innovación industrial; energía y minería; apropiación social del conocimiento; sociales y humanas; biotecnología y educación ( Ver Tabla 3).

Tabla 3. Total de programas y proyectos aprobados por PNCTel

Table 3. Total of programs and projects approved by PNCTeI

\begin{tabular}{|l|c|c|c|c|c|}
\hline \multicolumn{1}{|c|}{ PNCTel } & & \multicolumn{2}{c|}{ Vr. FCTel } & & \\
\hline & No. & VALOR & $\%$ & CONTRAP. & Vr. TOTAL \\
\hline Total general & $\mathbf{2 0 4}$ & $\mathbf{1 . 5 4 9 . 4 8 2}$ & $\mathbf{1 0 0 , 0 0 \%}$ & $\mathbf{4 4 5 . 4 1 0}$ & $\mathbf{1 . 9 9 4 . 8 9 2}$ \\
\hline PNCTel agropecuarias & 58 & 322.015 & $\mathbf{2 0 , 8 0 \%}$ & 124.168 & 446.183 \\
\hline Formación alto nivel & 21 & 300.744 & $19,40 \%$ & 26.139 & 326.882 \\
\hline Programa Ondas & 27 & 289.794 & $18,70 \%$ & 37.521 & 327.315 \\
\hline PNCTel en salud & 17 & 162.809 & $10,50 \%$ & 72.223 & 235.033 \\
\hline Electrónica, telecomunicaciones e informática & 9 & 79.923 & $5,20 \%$ & 33.246 & 113.169 \\
\hline PNCTel del Mar y de los recursos hidrobiológicos & 11 & 78.197 & $5,00 \%$ & 16.024 & 94.220 \\
\hline Centros y parques & 12 & 78.135 & $5,00 \%$ & 46.026 & 124.162 \\
\hline PNCTel en ambiente, biodiversidad y hábitat & 19 & 63.690 & $4,10 \%$ & 25.003 & 88.693 \\
\hline Desarrollo tecnológico e innovación industrial & 6 & 56.154 & $3,60 \%$ & 22.649 & 78.803 \\
\hline Investigaciones en energía y minería & 9 & 44.705 & $2,90 \%$ & 19.807 & 64.512 \\
\hline Apropiación social del conocimiento & 4 & 39.515 & $2,60 \%$ & 13.702 & 53.216 \\
\hline PNCTel de las áreas sociales y humanas & 5 & 18.171 & $1,20 \%$ & 5.197 & 23.368 \\
\hline Biotecnología & 4 & 11.669 & $0,80 \%$ & 3.689 & 15.358 \\
\hline PNCTel en educación & 2 & 3.961 & $0,30 \%$ & 17 & 3.978 \\
\hline
\end{tabular}

Fuente: Secretaría Técnica FCTel -SGR, Abril 01 de 2014 / Cifras en millones de pesos 
Programas y proyectos aprobados en el PNCTel agropecuarias

El sector agropecuario lidera la asignación de estos recursos de regalías, logrando el $20.8 \%$ del total de la inversión, con una aprobación de 58 proyectos en 22 departamentos, cuyo monto asciende a $\$ 322.015$ millones de pesos, distribuidos en temáticas como agroindustria, ganadería, hortofloricultura y café (Ver Fig. 1)

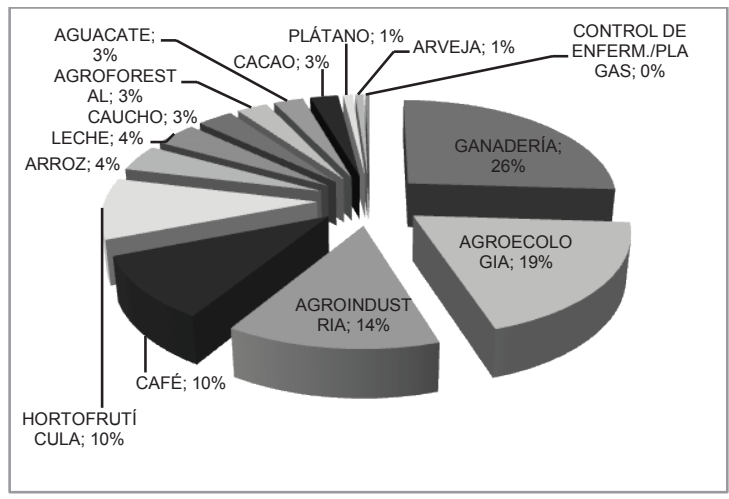

Figura 1. Programas y proyectos agropecuarios por temáticas

Figure 1. Programs and projects agricultural per topic Fuente: Secretaría Técnica FCTel-SGR, Abril 01 de 2014.

La región con más alta inversión es la Región Caribe con el $31 \%$ y la que menos Centro Oriente con el $7,2 \%$ del total de proyectos aprobados (Ver Fig. 2)

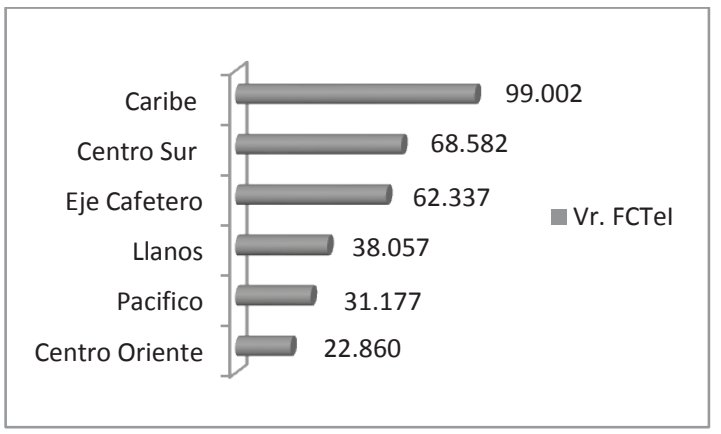

*Cifras en millones de pesos

Figura 2. Inversión por regiones en programas y proyectos agropecuarios por región

Figure 2. Investment per regions in agricultural programs and projects

Fuente: Secretaría Técnica FCTel-SGR, Abril 01 de 2014.
Aunque la mayor parte de los proyectos han sido presentados de forma individual por cada departamento, existen 2 programas y proyectos que han logrado la conformación de alianzas regionales, tales como "Estudio de sistemas de producción basado en la caracterización, tipificación y análisis de alternativas agropecuarias en tres zonas agroecológicas del departamento de Guainía», presentado por Guainía en alianza con Meta y Vichada y "Desarrollo de un programa de gestión tecnológica para la innovación social y productiva de la carne y la leche en sistemas de producción bovina de la región de los llanos en Colombia», presentado por Arauca en alianza con Vichada.

Por su parte, Antioquia participa con 12 proyectos del total de 58 proyectos aprobados en el país, cuyo monto solicitado al FCTel-SGR asciende a \$51.007 millones de pesos (Ver Fig. 3).

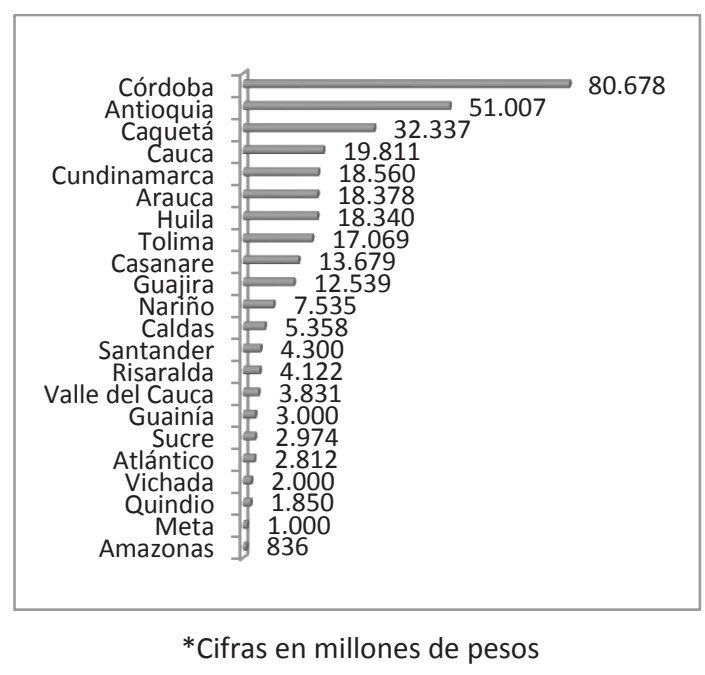

Figura 3. Inversión en programas y proyectos agropecuarios - distribución departamental

Figure 3. Investment in agricultural programs and projects - departmental distribution

Fuente: Secretaría Técnica FCTel-SGR, Abril 01 de 2014. 


\section{Los proyectos aprobados para Antioquia se presentan en la Tabla 4.}

Tabla 4. Programas y proyectos agropecuarios aprobados para Antioquia

Table 4. Agricultural programs and projects approved for Antioquia

\begin{tabular}{|l|l|}
\hline $\begin{array}{l}\text { Desarrollo tecnológico, productivo y comercial de } \\
\text { aguacate en Antioquia }\end{array}$ & $\begin{array}{l}\text { Fomento al cultivo de cacaos especiales en el } \\
\text { departamento de Antioquia }\end{array}$ \\
\hline $\begin{array}{l}\text { Fortalecimiento a los productores en el encadenamiento } \\
\text { productivo de fruta pequeña }\end{array}$ & $\begin{array}{l}\text { Fortalecimiento de la cadena de hortalizas en el } \\
\text { departamento de Antioquia a través de la generación de } \\
\text { un sistema de inocuidad y aprovechamiento de residuos } \\
\text { para potenciar suactividad }\end{array}$ \\
\hline $\begin{array}{l}\text { Fortalecimiento de la cadena productiva de leche del } \\
\text { distrito norte antioqueño }\end{array}$ & $\begin{array}{l}\text { Fortalecimiento del sector de las plantas aromáticas, } \\
\text { medicinales y condimentarias en el departamento de } \\
\text { Antioquia }\end{array}$ \\
\hline $\begin{array}{l}\text { Fortalecimiento proyecto Antioquia: origen de cafés } \\
\text { especiales en el departamento de Antioquia }\end{array}$ & $\begin{array}{l}\text { Investigación control caracol plaga achatina fulica y } \\
\text { disminución del riesgo en salud, agricultura y turismo con } \\
\text { la comunidad de Santafé de Antioquia }\end{array}$ \\
\hline $\begin{array}{l}\text { Investigación mejoramiento de la productividad del } \\
\text { cultivo del plátano suroeste y Urabá }\end{array}$ & $\begin{array}{l}\text { Investigación para el desarrollo de alternativas } \\
\text { productivas con especies promisorias de la biodiversidad } \\
\text { en el medio Atrato antioqueño }\end{array}$ \\
\hline $\begin{array}{l}\text { Investigación técnico-social de las oleaginosas promisorias } \\
\text { higuerilla y sacha inchi con miras a su desarrollo } \\
\text { agroindustrial }\end{array}$ & $\begin{array}{l}\text { Mejoramiento de la productividad para el desarrollo y } \\
\text { aumento en la competitividad en la cadena de caucho } \\
\text { natural, mediante un programa de investigación aplicada } \\
\text { en innovación en el departamento de Antioquia }\end{array}$ \\
\hline
\end{tabular}

\section{Programas y proyectos aprobados en formación de alto nivel}

Según la Guía Sectorial de Programas y Proyectos de Ciencia, Tecnología e Innovación (DNP, 2012), la actividad de Formación y capacitación científica y tecnológica, incluye la educación formal de capital humano para la CTel, el entrenamiento, la capacitación y la especialización de niños, jóvenes y profesionales interesados en el quehacer científico, en el diseño de políticas de ciencia y tecnología, y en la gestión del conocimiento y de la innovación (ver llustración 1)
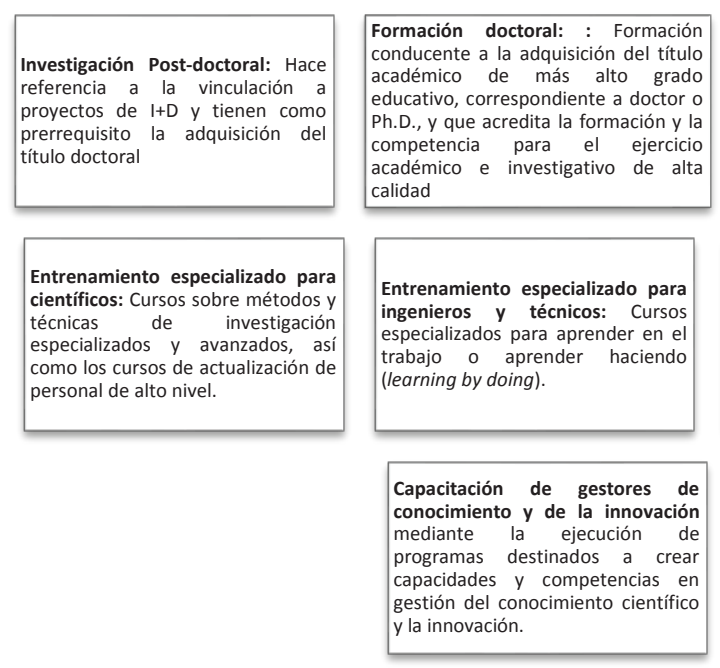

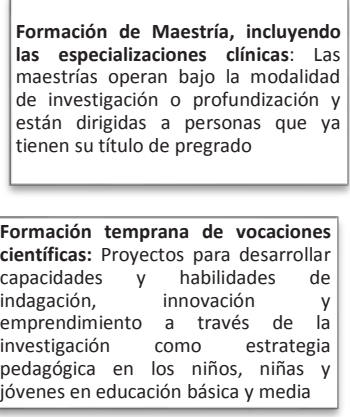

pedagogica en los niños, niñas y
jóvenes en educación básica y media

Ilustración 1. Actividades de formación y capacitación científica y tecnológica Graphic 1. Scientific and technological training

Fuente: Colciencias: Guía Sectorial de Programas y Proyectos de Ciencia, Tecnología e Innovación 
Al respecto, se destaca que la temática de formación de capital humano es la segunda en importancia respecto a la asignación de recursos del FCTel-SGR. El programa de formación de alto nivel tiene 21 proyectos en 18 departamentos y el Distrito Capital, por un valor aprobado de $\$ 300.744$ millones de pesos, que representa el $19.4 \%$ de la asignación total de recursos FCTel-SGR. De las 6 áreas de aplicación de los proyectos aprobados, el $69 \%$ de los recursos se destinan a financiar formación de doctorados y maestrías (ver Fig. 4).

Adicional a los proyectos de formación de alto nivel es importante evidenciar que los esfuerzos por formación de capital humano se encuentran vinculado en proyectos presentados en otros PNCTel, que involucran el componente de formación de alto nivel como un componente sustancial de los mismos.

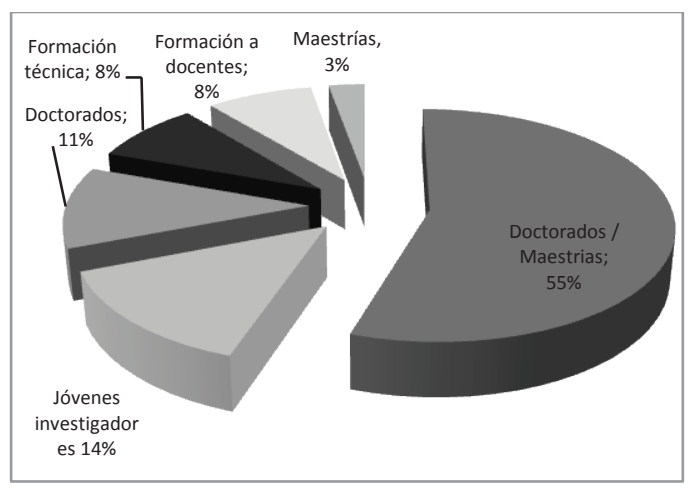

Figura 4. Programas y proyectos por nivel de formación Figure 4. Programs and projects per level of training

Fuente: Secretaría Técnica FCTel-SGR, Abril 01 de 2014.

Al respecto, se encuentra que el $16 \%$ de las becas de maestría y doctorado son financiadas por PNCTel, diferentes al Programa de formación de alto nivel (Ver Tabla 5).

Tabla 5. Programas y proyectos de formación alto nivel en otros PNCTel Table 5. Other Phd and Master training programs

\begin{tabular}{|c|l|c|c|c|}
\hline \multicolumn{2}{|c|}{ Resultados Esperados } & $\begin{array}{c}\text { Proyectos de Formación } \\
\text { de alto nivel } \\
\text { Tipo de formación }\end{array}$ & $\begin{array}{c}\text { Otros Proyectos con } \\
\text { componente formación }\end{array}$ & \multirow{2}{*}{ Total } \\
\hline \multirow{2}{*}{ Beneficiarios de becas } & Beneficiarios de becas & \\
\hline \multirow{2}{*}{ Maestrías } & Nacionales & 1560 & 268 & 1828 \\
\cline { 2 - 5 } & Internacionales & 177 & 16 & 193 \\
\hline \multirow{2}{*}{ Doctorados } & Nacionales & 261 & 111 & 372 \\
\cline { 2 - 5 } & Internacionales & 125 & 6 & 131 \\
\cline { 2 - 5 } & Inserción laboral & 37 & 4 & 41 \\
\hline & Total & $\mathbf{2 1 6 0}$ & $\mathbf{4 0 5}$ & $\mathbf{2 5 6 5}$ \\
\hline
\end{tabular}

Fuente: Secretaría Técnica FCTel -SGR, 01 de abril de 2014.

A nivel territorial, se observa que todas las regiones participan en el programa de formación de alto nivel, siendo la región Caribe la región que más aporta recursos del
FCTel-SGR con un $42 \%$, y la que menos registra recursos en este aspecto es la región Centro Sur con 5\% (Ver Fig. 5). 


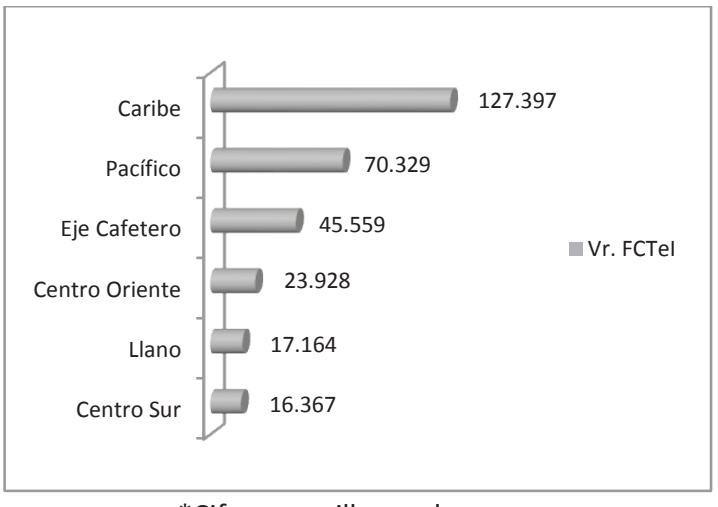

*Cifras en millones de pesos

Figura 5. Inversión en programas y proyectos de formación de alto nivel por región

Figure 5. Investment in Ph.D. and Master programs and projects per region

Fuente: Secretaría Técnica FCTel -SGR, Abril 01 de 2014.

En los proyectos de formación de alto nivel, Antioquia participa con un (1) proyecto de formación del total de los 31 proyectos aprobados en el país, y cuyo monto solicitado al FCTel-SGR asciende a \$23.500 millones de pesos. El proyecto Capacitación mediante becas de maestría a docentes de establecimientos educativos de los municipios no certificados en el departamento de Antioquia, tiene como objetivo mejorar los niveles de formación avanzada de los docentes de los municipios no certificados en educación de Antioquia, a través del acceso a becas de maestría en diferentes áreas del conocimiento. Estas becas están enfocadas en los componentes de ciencia, tecnología e innovación, integrados a las áreas básicas de la educación y gestión institucional (Matemáticas, Lengua Castellana, Ciencias Naturales, Tecnología e Informática e Inglés) (SEDUCA, 2013).
Programas y proyectos aprobados en el programa Ondas

El programa Ondas es la estrategia fundamental de Colciencias para el fomento de una cultura ciudadana y democrática en CTel en la población infantil y juvenil colombiana, a través de la Investigación como Estrategia Pedagógica-IEP.

Este programa cuenta con 27 proyectos en 24 departamentos (tres departamentos, Córdoba, Meta y Santander, cuentan con dos proyectos aprobados en este programa, un es con énfasis básico en Ondas, y el otro es Ondas apoyado en TIC), por un valor de \$289.794 millones de pesos, representando el $18.7 \%$ de la asignación de los recursos. Las principales áreas de aplicación de los proyectos aprobados son Pedagogía, Ondas TIC, Emprendimiento, Software y Robótica (Ver Fig. 6)

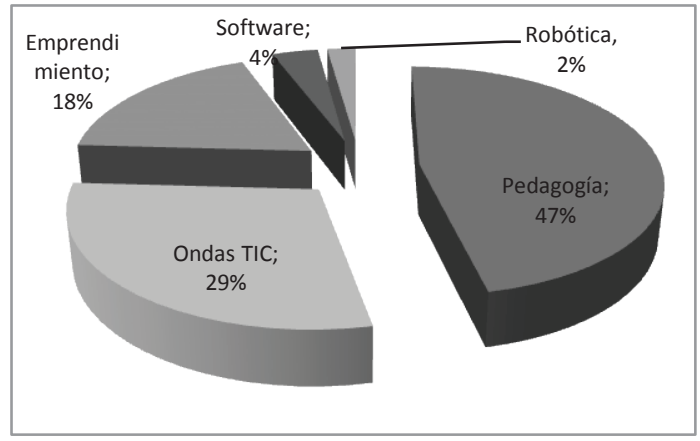

Figura 6. Programas y proyectos del programa Ondas por áreas

Figure 6. Ondas programs and projects per areas

Fuente: Secretaría Técnica FCTel -SGR, Abril 01 de 2014.

Todas las regiones participan en el programa. La región que más aporta recursos del FCTelSGR es el Centro Oriente (30\%), y la que menos Eje Cafetero (6\%) (Ver Fig. 7); Antioquia no cuenta con proyectos aprobados en esta área. 


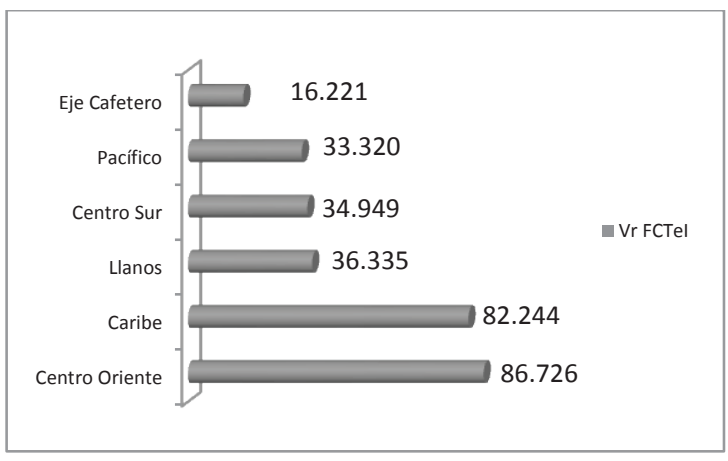

*Cifras en millones de pesos

Figura 7. Inversión por regiones en programas y proyectos del programa Ondas por región

Figure 7. Investment per region in Ondas programs and projects

Fuente: Secretaría Técnica FCTel -SGR, Abril 01 de 2014.

Programas y proyectos aprobados en el programa de salud

En el programa de $\mathrm{CT}+\mathrm{I}$ en salud, se han aprobado 15 proyectos en 11 departamentos por \$158.621 millones de pesos, representando el $10.5 \%$ de la asignación de los recursos. Las principales áreas vinculadas en los proyectos son telemedicina, enfermedades tropicales y farmacología, entre otras áreas (Fig. 8).

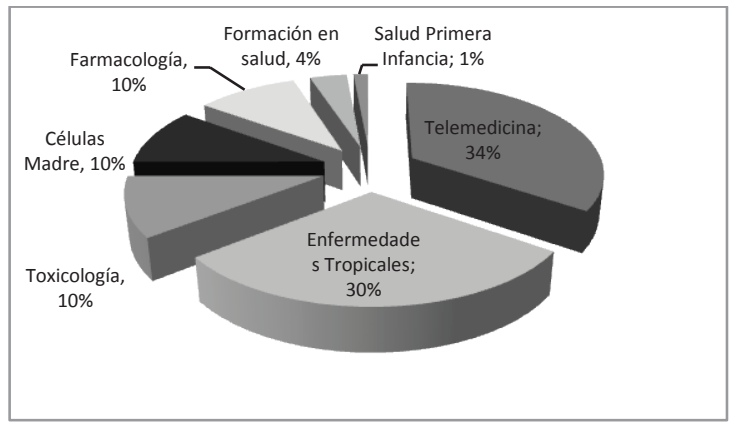

Figura 8. Programas y proyectos del programa de salud por áreas

Figure 8. Health programs and projects per areas

Fuente: Secretaría Técnica FCTel -SGR, Abril 01 de 2014.

Aunque todas las regiones tienen participación en esta área, la región con más alta inversión es el Eje Cafetero con el 47\%, y la que menos, Región Pacífica con el 1\% del total de proyectos aprobados (Ver Figura 9).

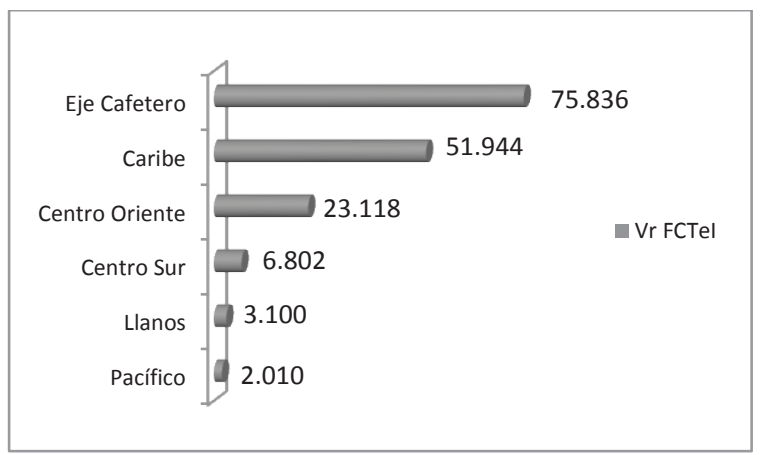

*Cifras en millones de pesos

Figura 9. Inversión por regiones en programas y

proyectos en el programa de salud por región

Figure 9. Investment per region in health programs and projects

Fuente: Secretaría Técnica FCTel -SGR, Abril 01 de 2014.

Antioquia lidera la inversión en proyectos y programas en Salud, participando con 4 proyectos del total de 15 proyectos aprobados en el país, cuyo monto solicitado al FCTel asciende a \$71.545 millones de pesos (Ver Fig. 10).

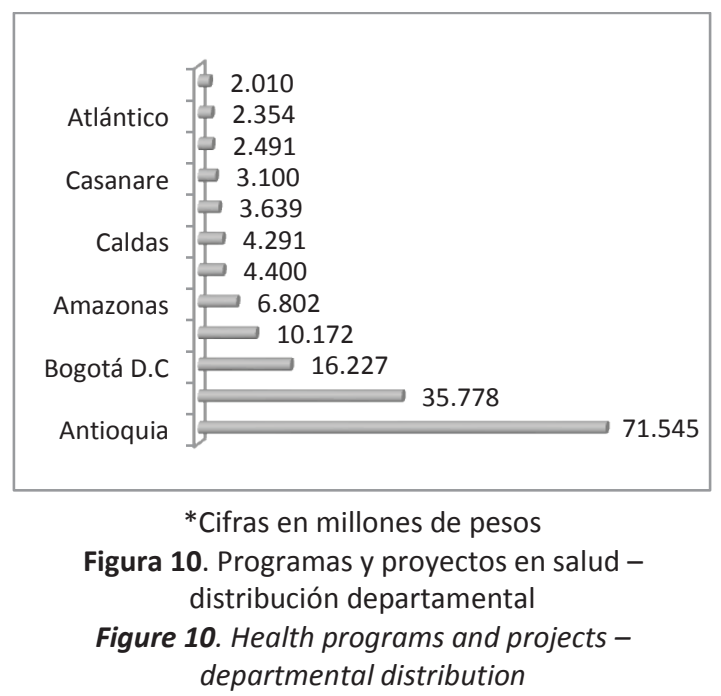

Fuente: Secretaría Técnica FCTel -SGR, Abril 01 de 2014.

Los proyectos aprobados para Antioquia en este programa se presentan en la Tabla 7. 
Tabla 7. Programas y proyectos aprobados para Antioquia

Table 7. Programs and projects approved for Antioquia

Desarrollo de nuevos agentes terapéuticos para el tratamiento de enfermedades de importancia en salud pública en Antioquia

Desarrollo de soluciones en ciencia, tecnología e innovación - CTI para el sector salud en Antioquia

Desarrollo de soluciones en ciencia, tecnología e innovación para el asesoramiento especializado en farmacología y toxicología en Medellín, Antioquia

Desarrollo de soluciones en CTI para Telesalud en el departamento de Antioquia

Programas y proyectos aprobados en el programa de Electrónica, Telecomunicaciones e Informática -ETI-

El programa Electrónica, Telecomunicaciones e Informática -ETI, participa con 9 proyectos en 7 departamentos por $\$ 79.923$ millones de pesos FCTel-SGR, representando el $5.2 \%$ de la asignación de los recursos. De las 5 áreas de aplicación de los proyectos aprobados, el $55 \%$ de los recursos se destinan a financiar temáticas relacionadas con emprendimiento. Ver Fig. 11.

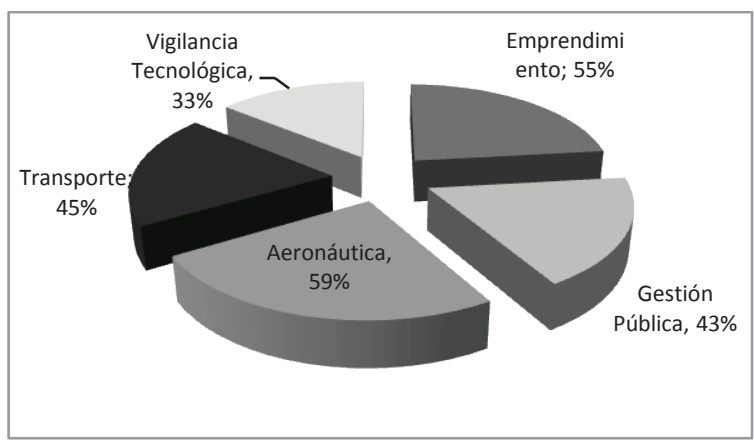

Figura 11. Programas y proyectos del programa ETI por áreas

Figure 11. Programs and projects in electronic, telecommunication and information per area

Fuente: Secretaría Técnica FCTel -SGR, Abril 01 de 2014.
La región con más alta inversión es el Pacífico con el $44 \%$, y la región con menos inversión es Centro Sur con el $2 \%$ del total de proyectos aprobados (Ver Figura 12).

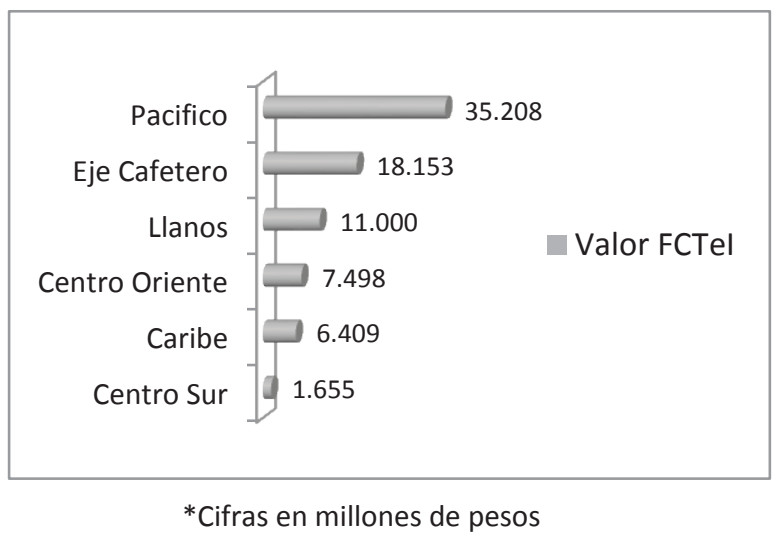

Figura 12. Inversión por regiones en programas y proyectos en el programa ETI

Figure 12. Investment per regions in electronic, telecommunication and information programs and projects

Fuente: Secretaría Técnica FCTel -SGR, Abril 01 de 2014.

Aunque la mayor parte de los proyectos han sido presentados de forma individual por cada departamento, existe un proyecto que ha logrado la conformación de alianzas regionales entre departamentos de distintas regiones del país. El proyecto Desarrollo Investigación aplicada para contribuir a un modelo efectivo y sostenible de intervención del dengue en Santander, Casanare y Valle del Cauca, tiene una dimensión intrarregional, al articular departamentos de la región Centro Oriente, Llanos y Pacífico.

Por su parte, Antioquia participa con (1) un proyecto del total de 9 proyectos aprobados en el país, cuyo monto solicitado al FCTelSGR asciende a $\$ 5.000$ millones de pesos (Ver Figura 13). El proyecto aprobado para Antioquia es Estudio, selección y estandarización de sistemas tecnológicos alternativos para rehabilitación y tratamiento de puntos críticos en vías y desarrollo de pruebas piloto en subregiones de Antioquia, con el cual se busca el 
desarrollo, implementación y análisis de una canasta de sistemas tecnológicos alternativos para la intervención de la red vial secundarias en tres objetivos: estabilización de taludes, estabilización de rasantes y protección y prevención de la erosión en taludes.

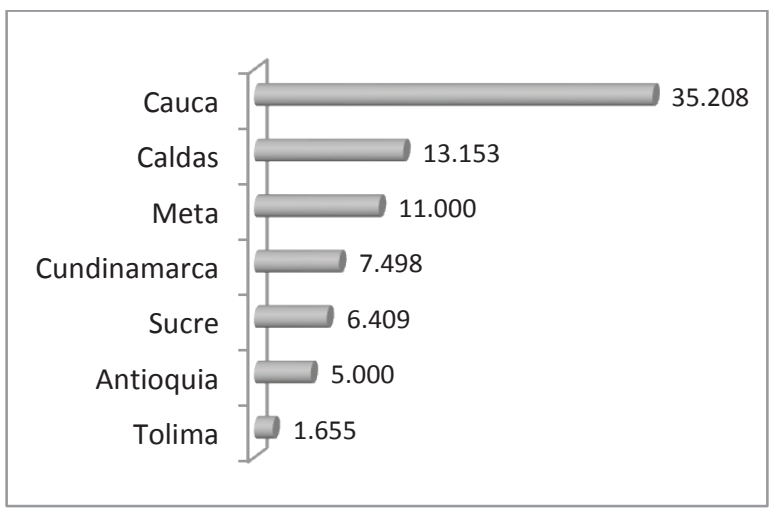

*Cifras en millones de pesos

Figura 13. Programas y proyectos en el programa ETI distribución departamental

Figure 13. Programs and projects in electronic, telecommunication and information-departamental distribution

Fuente: Secretaría Técnica FCTel -SGR, Abril 01 de 2014.

Programas y proyectos aprobados en el programa Mar y Recursos Hidrobiológicos

El programa de Mar y Recursos Hidrobiológicos participa con 11 proyectos en 9 departamentos por un total de $\$ 78.197$ millones de pesos del FCTel, representando el 5.0\% de la asignación de los recursos. Las principales áreas de aplicación de los proyectos aprobados son recursos hídricos, acuicultura y logística de puertos (Fig.14).

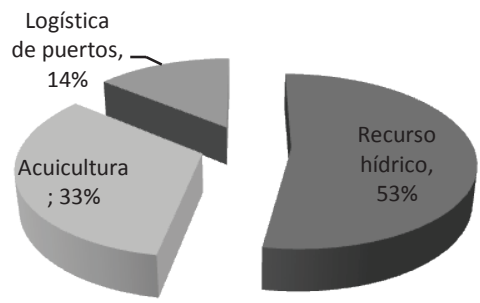

Figura 14. Programas y proyectos del programa Mar y Recursos Hidrobiológicos por áreas

Figure 14. Sea and hydrobiological resources programs and projects per areas

Fuente: Secretaría Técnica FCTel -SGR, Abril 01 de 2014.

La región que más aporta recursos del FCTelSGR al programa de Mar y Recursos Hidrobiológicos es la Región Caribe (77\%), y la región que menos es Llanos (1\%) (Ver Fig. 15). En este programa se presentan dos proyectos en los cuales se cuenta con la articulación de varios departamentos de la Región Caribe. Estos proyectos son: i) Investigación Innovación en Logística y Puertos: LOGPORT Caribe, en el cual participan Atlántico, Bolívar y Sucre y ii) Desarrollo sostenible de la acuicultura en el Caribe colombiano, en el cual participan Bolívar, Guajira y Sucre.

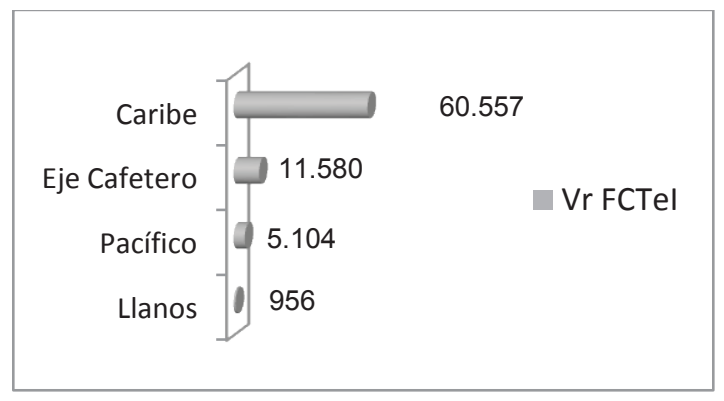

*Cifras en millones de pesos

Figura 15. Inversión por regiones en programas y proyectos del programa Mar y Recursos Hidrobiológicos

Figure 15. Programs and projects sea and hydrobiological resources investment per regions Fuente: Secretaría Técnica FCTel -SGR, Abril 01 de 2014. 
Antioquia participa con (1) un proyecto del total de 11 proyectos aprobados en el país, cuyo monto solicitado al FCTel asciende a $\$ 11.580$ millones de pesos (Ver Fig. 16). El proyecto aprobado para Antioquia es Fortalecimiento regional de la pesca y la acuicultura en Antioquia.

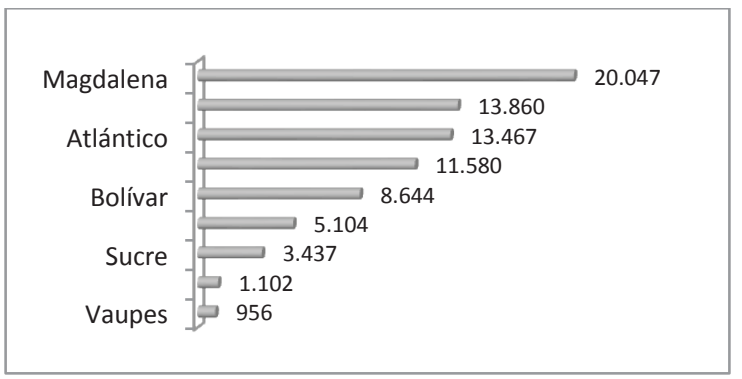

*Cifras en millones de pesos

Figura 16 Programas y proyectos en el programa Mar y Recursos Hidrobiológicos- distribución departamental

Figure 16. Programs and projects sea and hydrobiological resources - departamental distribution

Fuente: Secretaría Técnica FCTel-SGR, Abril 01 de 2014.

\section{Programas y proyectos aprobados en el PNCTel Centros y Parques}

El programa de centros y parques tecnológicos participa con 12 proyectos en 9 departamentos por $\$ 78.135$ millones de pesos FCTel-SGR, representando el $5.0 \%$ de la asignación de los recursos del Fondo. De las 2 áreas de aplicación de los proyectos aprobados el $57 \%$ de los recursos se destinaron a financiar parques tecnológicos, y el otro $43 \%$ a financiar centros de investigación.

La región que más aporta recursos del FCTelSGR al programa centros y parques es la región de Centro Oriente (36\%), y la región que menos aporta recursos a este programa es la Región Llanos (2\%) (Ver Fig.17).

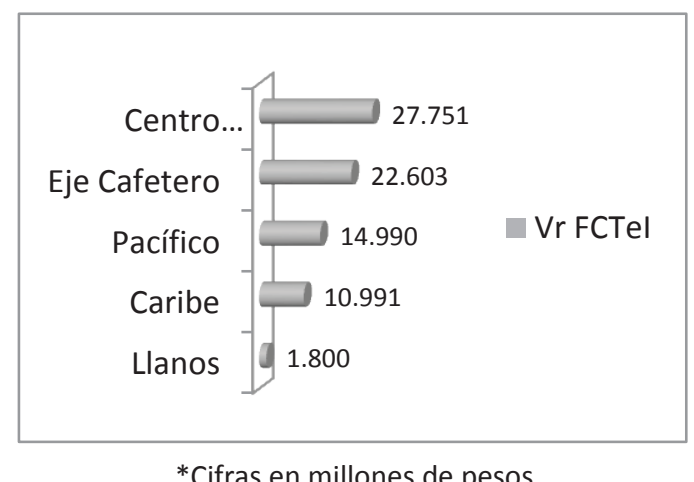

Figura 17 Inversión por regiones en centros y parques tecnológicos

Figure 17. Technological parks and R\&D institutions Investment per region

Fuente: Secretaría Técnica FCTel -SGR, Abril 01 de 2014.

Dentro de los parques tecnológicos apoyados se encuentran: Parque Tecnológico del Caribe, Parque Científico de Cundinamarca, City Tech, Parque Tecnológico de innovación para la agregación de valor a la caficultura caucana, Parque Tecnológico del Sinú y San Jorge en el departamento de Córdoba, y el Parque Tecnológico de Guatiguara ( Ver Tabla 8 ). 
Tabla 8. Programas y proyectos aprobados para el PNCTel de Centros y Parques

Table 8. Programs and projects approved for technological parks and R\&D institutions

1. Desarrollo estudios y capacidades de gestión tecnológica para la puesta en marcha del parque tecnológico del caribe todo el departamento, Atlántico, Caribe

Caldas

1. Implementación creación y dotación de la infraestructura física y tecnológica de City Tech como parque tecnológico región centro surdepartamento de Caldas

2. Implementación del centro de investigación, innovación y tecnología al sector panelero del departamento de Caldas (centro BEKDAU) Supía, Caldas.

\section{Cauca}

1. Consolidación de un centro de investigación, promoción e innovación social para el desarrollo de la caficultura caucana

2. Implementación y conformación del parque tecnológico de innovación para la agregación de valor a la caficultura caucana

\section{Córdoba}

1. Estudio de factibilidad del parque tecnológico del Sinú y san Jorge en el departamento de Córdoba

\section{Cundinamarca}

1. Fortalecimiento capacidades de innovación social por medio del Parque Científico Cundinamarca y Bogotá

\section{Risaralda}

1. Consolidación del centro de innovación y desarrollo tecnológico de la manufactura y la metalmecánica -CINDETEMM Dosquebradas, Risaralda

2. Implementación del centro de innovación y desarrollo tecnológico con enfoque de gestión en KPO Pereira, Risaralda

\section{Meta}

1. Estudio de factibilidad para la creación del centro de investigación y desarrollo tecnológico de la región de los llanos, Meta, Orinoquía

\section{Santander}

1. Fortalecimiento de los procesos de transferencia tecnológica en el parque tecnológico de Guatiguara

\section{Sucre}

1. Instituto investigaciones biomédicas del Caribe

\section{Balance del estado Actual de Antioquia en proyectos aprobados por el FCTel-SGR.}

Del total de los 204 proyectos aprobados, Antioquia participa con 26 proyectos que suman \$175.781 millones de pesos del FCTelSGR, liderando los montos de inversión en este Fondo.
Antioquia ha enfocado sus procesos de fortalecimiento de la CTel, principalmente en los sectores de la salud, con 5 proyectos que representan el $41 \%$ del total de los recursos aprobados para el departamento; el sector agropecuario que participa con 12 proyectos que representan el $29 \%$ de los recursos aprobados y la formación de alto nivel con un proyecto, logrando el $13 \%$ de la inversión (Tabla 9). 
Tabla 9. Programas y proyectos aprobados por PNCTel - ANTIOQUIA

Table 9. Programs and projects approved for PNCTeI - ANTIOQUIA

\begin{tabular}{|l|c|c|c|c|c|}
\hline \multicolumn{1}{|c|}{ PNCTel } & No. & FCTel & $\%$ & CONTRAP. & Vr. TOTAL \\
\hline Total general & $\mathbf{2 6}$ & $\mathbf{1 7 5 . 7 8 1}$ & $\mathbf{1 0 0 \%}$ & $\mathbf{1 0 6 . 9 8 3}$ & $\mathbf{2 8 2 . 7 6 4}$ \\
\hline CTel salud & 5 & 71.545 & $41 \%$ & 41.190 & 112.735 \\
\hline CTel agropecuarias & 12 & 51.007 & $29 \%$ & 54.302 & 105.309 \\
\hline Formación alto nivel & 1 & 23.500 & $13 \%$ & 2.000 & 25.500 \\
\hline CTel Mar y de los Rec. Hidrobiológicos & 1 & 11.580 & $7 \%$ & 3.496 & 15.076 \\
\hline ETI & 1 & 5.000 & $3 \%$ & 414 & 5.414 \\
\hline Investigación en energía y minería & 2 & 4.539 & $3 \%$ & 3.938 & 8.477 \\
\hline CTel áreas Sociales Y Humanas & 1 & 3.663 & $2 \%$ & 688 & 4.351 \\
\hline CTel Educación & 1 & 2.930 & $2 \%$ & 0 & 2.930 \\
\hline Ctel Ambiente, biodiversidad y hábitat & 2 & 2.017 & $1 \%$ & 954 & 2.972 \\
\hline
\end{tabular}

Fuente: Secretaría Técnica FCTel -SGR, Abril 01 de 2014 / Cifras en millones de pesos

\section{CONCLUSIONES}

La entrada en vigencia del SGR y la respectiva creación del Fondo de CTel ha abierto una oportunidad para la CTel en el país, por cuanto los recursos asignados están casi que triplicando el actual presupuesto de inversión de Colciencias en programas y proyectos de CTel, que es de $\$ 550.592$ millones. Se espera que los nuevos recursos impacten a las regiones no solo en el corto sino también en el mediano y largo plazo.

La inversión de los recursos presenta un reto importante en lo referente con la articulación de redes y alianzas entre regiones y actores del Sistema Nacional de Ciencia, Tecnología e Innovación, para invertir estos recursos de acuerdo con las apuestas planteadas en los planes de desarrollo y los planes departamentales de ciencia, tecnología e innovación. La mayoría de proyectos aprobados han sido de carácter departamental, a pesar de que uno de los objetivos del FCTel es generar alto impacto regional.

Las regiones han orientado su estrategia de inversión hacia dos objetivos: por una parte, atender problemas específicos a través de actividades de investigación e innovación; por otra, las regiones le han apostado a la formación de capital humano como una estrategia de mediano y largo plazo, capital que podrá posteriormente elevar las capacidades de investigación e innovación en las regiones.

El liderazgo que ha tenido Antioquia en la aprobación de proyectos de CTel, además de destacarse el número de proyectos aprobados, también lo hace la diversificación de temas estratégicos priorizados para presentar al FCTel-SGR, enfocando los esfuerzos en el fortalecimiento de la CTel en los sectores de la salud, agropecuario y formación de capital humano, entre otros.

\section{REFERENCIAS}

COLCIENCIAS. Programa Ondas. Recuperado de:

http://www.colciencias.gov.co/progr ama_estrategia/programa-ondas.

Departamento Nacional de Planeación (2012). Manual metodológico general, para la identificación, preparación, programación y evaluación de proyectos. Recuperado 


\author{
de: \\ http://www.colciencias.gov.co/sites/ \\ default/files/ckeditor_files/files/7_\% \\ 20Documento\%20Guia\%20Sectorial- \\ FCTel.pdf \\ Departamento Nacional de Planeación \\ (2007). Actualización de la cartilla \\ "Las regalías en Colombia». \\ Recuperado de: \\ https://www.dnp.gov.co/Portals/0/a \\ rchivos/documentos/DR/Regalias_Pu \\ blicaciones_Prensa/Cartilla_las_regal \\ \%C3\%ADas_en_colombia2008.pdf
}

\author{
Ministerio de Minas y Energía (2011). \\ Memorias al Congreso de la \\ República 2010-2011: Regalías. \\ Recuperado de: \\ http://www.minminas.gov.co/minmi \\ nas/downloads/UserFiles/File/Memo \\ rias/Memorias_2011/02- \\ REGALIAS.pdf \\ Seduca (2013). Becas de maestría para \\ docentes en Antioquia! \\ (Convocatoria).Recuperado de: \\ http://www.seduca.gov.co/index.ph \\ p/sala-de-prensa/2905-800-becas- \\ de-maestrias-profes-antioquia- \\ .html\#.U3OYHXb5T1U
}

